To appear in Lecture Notes in Computational Science and Engineering (LNCSE),

Springer-Verlag Publishing, 2006

\title{
Space-Time Error Representation and Estimation in Navier-Stokes Calculations
}

\author{
Timothy J. Barth \\ NASA Ames Research Center, Moffett Field, CA 94035 USA \\ Timothy.J.Barth@nasa.gov
}

Summary. The mathematical framework for a-posteriori error estimation of functionals elucidated by Eriksson et al. [7] and Becker and Rannacher [3] is revisited in a space-time context. Using these theories, a hierarchy of exact and approximate error representation formulas are presented for use in error estimation and mesh adaptivity. Numerical space-time results for simple model problems as well as compressible Navier-Stokes flow at $R e=300$ over a $2 \mathrm{D}$ circular cylinder are then presented to demonstrate elements of the error representation theory for time-dependent problems.

\section{Introduction}

For better or worse, our physical world is constantly evolving in time. Many important physical phenomena depend fundamentally on time either deterministically or through dynamical system behavior. As the complexity of numerical time-dependent fluid flow simulations continues to rapidly increase with advancements in computer hardware, the ability to represent, estimate and control numerical errors occurring in these time-dependent simulations becomes paramount so that the uncertainty and risk associated with using these results in engineering designs becomes acceptable. This task is especially difficult for systems such as the Navier-Stokes equations where the prospect of controlling pointwise errors is intractable at sufficiently large Reynolds numbers. In this latter case, a pointwise error bound exists but the bound grows too rapidly with Reynolds number thus rendering the result useless. Even so, it still may be feasible to represent, estimate, and control (via adaptivity) the error in certain space and space-time integrated quantities such as body forces, averages, and other fluid statistics that can mathematically represented as functionals, the mapping of a function space to a single real number. In these special cases, the integration process "averages out" the sensitivity of pointwise errors to solution perturbations so that useful error bounds are obtained. For a given solution data, 
$u$, in $m$ dependent variables, these scalar output functionals will be denoted by $J(u): \mathbb{R}^{m} \mapsto \mathbb{R}$. For example, an output functional considered in the next section is the space-time average of the $i$-th solution component in the hypercube centered at $\left(x_{0}, y_{0}, z_{0}, t_{0}\right)$

$$
J\left(u_{i}\right)=\int_{t_{0}}^{t_{1}} \int_{d \times d \times d} u_{i}\left(x-x_{0}, y-y_{0}, z-z_{0}, t-t_{0}\right) d x^{3} d t
$$

\subsection{Computability of Functional Outputs}

To illustrate the benefits of estimating integrated functional outputs, Hoffman and Johnson [14] have computed solutions of the backward facing step problem shown in Fig. 1 at $R e=2000$ using a conforming finite element method with linear elements for incompressible flow, see [14] for details. Space-time error estimates were then constructed for the functional (1) for various values of the box width $d$ together with the time averaging interval $t_{0}=9, t_{1}=10$. In velocity and pressure variables

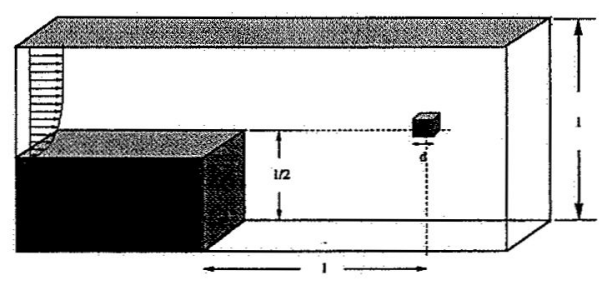

Fig. 1. Schematic of backward facing step problem showing $J(u)$ averaging box position used by Hoffman and Johnson.

$(V, p)$, the following error estimate for the streamwise velocity component functional have been obtained in terms of the backwards in time dual solution $(\psi, \phi)$ and the element residuals $r_{h, 0}$ and $r_{h, 1}$ for the $(V, p)$ system

•

$$
\begin{aligned}
\left|J(V, p)-J\left(V_{h}, p_{h}\right)\right| & \leq C_{0}\|\dot{\psi}\|\left\|h_{0} r_{h, 0}\left(V_{h}, p_{h}\right)\right\|+C_{1}\left\|D^{2} \phi\right\|\left\|h^{2} r_{h, 0}\left(V_{h}, p_{h}\right)\right\| \\
& +C_{2}\|\dot{\phi}\|\left\|h_{0} r_{h, 1}\left(V_{h}, p_{h}\right)\right\|+C_{3}\|D \phi\|\left\|h r_{h, 1}\left(V_{h}, p_{h}\right)\right\|
\end{aligned}
$$

where $h_{0}$ and $h$ are the temporal and spatial element lengths. Motivated by this estimate, stability factors for boxwidths $d=1 / 8,1 / 4,1 / 2$ have been computed by Hoffman and Johnson as summarized in Table 1.

The growth in stability factor magnitude with decreasing boxwidth size clearly shows a deterioration in the error estimate for the functional (1) as a pointwise error estimate is approached. In that same work, Hoffman and Johnson also use a drag force functional for incompressible flow over a cube geometry attached to a solid wall. By using error estimates of the drag functional in mesh adaptation, the mesh is only locally refined in a small portion of the domain so that numerical errors in the drag force are controlled using a relatively small number of degrees of freedom. For other 


\begin{tabular}{|c|c|c||c|c|}
\hline $\mathrm{d}$ & $\|\psi\|$ & $\|\nabla \psi\|$ & $\|\nabla \phi\|$ & $\|\phi\|$ \\
\hline $1 / 8$ & 124.0 & 836.0 & 138.4 & 278.4 \\
$1 / 4$ & 39.0 & 533.4 & 48.9 & 46.0 \\
$1 / 2$ & 10.5 & 220.3 & 16.1 & 25.2 \\
\hline
\end{tabular}

Table 1. Stability factors for the backward facing step problem for the averaging functional (1) for $d=1 / 8,1 / 4,1 / 2$.

problems, the overall challenge is not only to find convenient ways to estimate and control the numerical error in these functionals, but also to find suitable functionals that are both physically meaningful and lead to the resolution of the essential physics in the simulation.

\subsection{Estimation of Functional Outputs}

Let $u$ denote the exact solution data for a given problem and $u_{h}$ a numerical approximation of this exact solution. The task at hand is the representation, estimation, and control of errors in the numerically approximated functional so that given a user specified tolerance TOL

$$
\left|J(u)-J\left(u_{h}\right)\right|<\text { TOL }
$$

is achieved using minimal resources.

At first glance, the inclusion of time derivatives into the formulation appears to significantly complicate the construction of error bounds for functionals. Fortunately, for the class of uniform space-time finite-element methods this is not the case since the time dimension is discretized in precisely the same way that any spatial dimension is discretized. Specifically, we consider herein a space-time variant of the discontinuous Galerkin (DG) method due to Reed and Hill [24] and LeSaint and Raviart [19] for hyperbolic problems. In the space-time DG. method, the finite element discretization in time naturally yields time causality so that discretizations can be marched forward in time on a time-slab by time-slab basis for both hyperbolic and parabolic problems. Thus conceptually, the addition of time into the DG method does not introduce any new analysis complications. As a practical matter, full space-time formulations of DG are quite computationally expensive but extremely accurate methods.

\subsection{Preview of Space-Time Error Bounds}

For an introduction to a posteriori error analysis of functionals see the articles by Eriksson et al. [7], Becker and Rannacher [3], Giles et al. [9, 10], Johnson et al. [17], Prudhomme and Oden [22, 23], Süli [25], the collected NATO lecture notes [1] and the multitude of additional references contained therein. In Sect. 2, a brief introduction to these general theories is given with special attention given to space-time generalizations of previous results for the discontinuous Galerkin method previously given in Larson and Barth [18] as well as Hartmann and Houston [13]. 
Our main goal is to present the formulation of error estimates for output functionals in space-time. To illustrate the general form of these estimates, consider the space-time hyperbolic problem with initial data $u_{0}(x)$ and space-time boundary data $g(x, t)$ :

$$
\begin{aligned}
\mathcal{L} u-s(x, t) \equiv u_{, t}+\operatorname{div}(f(u))-s(x, t) & =0, & & \text { in } \Omega \times[0, T] \\
p^{-}(u-g(x, t)) & =0, & & \text { on } \Gamma \times[0, T] \\
u(x, t=0)-u_{0}(x) & =0, & & \text { initial data }
\end{aligned}
$$

where $p^{ \pm}$is a characteristic space-time projector such that $p^{-}=1$ at space-time inflow and $p^{+}=0$ at space-time outflow. Let $\mathcal{K}$ be a partition of a polygonal domain $\Omega$ with spatial boundary $\Gamma$ into non-overlapping shape regular elements (or control volumes) denoted by $K$, let $I^{n}$ denote an interval in time $I^{n} \equiv\left[t_{+}^{n}, t_{-}^{n+1}\right]$, and $Q^{n} \equiv K \times I^{n}$ a single space-time element with space-time dimension $h$. Throughout, we always consider time integration to a fixed final time $T$ using $N$ discrete time slabs such that $[0, T]=\cup_{n=0}^{N-1} \bar{I}^{n}$. Further, let $(a, b)_{Q} \equiv \int_{Q}(a \cdot b) d x d t$ and $\langle a, b\rangle_{\partial Q} \equiv \int_{\partial Q}(a \cdot b) d x d t$. In Sect. 2, the abstract theory is given for space-time error representation and estimation of functionals which has historically served as the basis for constructing weighted and unweighted estimates of the form (trivially extended here to include time):

- Weighted error estimates for functionals, Becker and Rannacher [3]

$$
\left|J(u)-J\left(u_{h}\right)\right| \leq \sum_{n=0}^{N} \sum_{Q^{n}}\left|\left(r_{h}, \phi-\pi_{h} \phi\right)_{Q^{n}}+\sum_{\partial Q^{n}}\left\langle j_{h}, \phi-\pi_{h} \phi\right\rangle_{\partial Q^{n}}\right|
$$

where $r_{h}$ denotes the residual on element interiors

$$
r_{h} \equiv \mathcal{L} u_{h}-s(x, t)
$$

Similariy, $j_{h}$ denotes an interface residual for a space-time element, $Q^{n} \equiv K \times I^{n}$, due to jumps occurring at element interfaces and inflow boundaries, viz.

$$
j_{h} \equiv\left\{\begin{array}{cc}
p^{-}\left[f\left(u_{h}\right) \cdot n\right]_{x_{-}^{n}}^{x_{n}^{n}}, & \partial K \backslash \Gamma \times I^{n} \\
p^{-}\left(f(g) \cdot n-f\left(u_{h}\right) \cdot n\right), & \partial K \cap \Gamma \times I^{n} \\
{\left[u_{h}\right]_{t_{+}^{n}}^{t_{n}^{n}},} & K, n \neq 0 \\
\left(u_{h}\left(t_{+}^{0}\right)-u_{0}(x)\right), & K, n=0
\end{array}\right.
$$

In the basic error estimate, $\phi$ represents an auxiliary function obtained from solving a (backwards in time) dual problem and $\pi_{h} \phi$ is any projection of $\phi$ into the approximation space containing $u_{h}$ as discussed in Sect. 2. Suppose $J(u)=$ $\int_{\Omega \times[0, T]}(\Psi \cdot u) d x d t$ for a sufficiently smooth weighting function $\Psi$, then the dual problem is related to the locally linearized adjoint problem

$$
\mathcal{L}^{*} \phi=\Psi
$$

together with appropriate boundary conditions. Observe that the functional error is a weighted combination of numerical residuals with weighting function $\phi-\pi_{h} \phi$. 
- Error estimates for functionals via stability factors, Johnson et al. [17, 14]. The computation of the weighted error estimate requires the resolution of a dual problem $\phi$ which becomes computationally expensive when performed in spacetime. In the unweighted estimate of Johnson et al. [17], basic approximation theory results are applied to the dual problem appearing in (5) thus yielding

$$
\left|J(u)-J\left(u_{h}\right)\right| \leq C_{0}\|\phi\|_{H^{s}(\Omega \times[0, T])}\left(\sum_{n=0}^{N-1} \sum_{Q^{n}}\left(h^{s}\left\|r_{h}\right\|_{Q^{n}}+h^{s-1 / 2}\left\|j_{h}\right\|_{\partial Q^{n}}\right)^{2}\right)
$$

for $1 \leq s \leq p+1$. The dual problem is then replaced with a stability constant satisfying

$$
\|\phi\|_{H^{s}(\Omega \times[0, T])} \leq C_{\text {stab }}
$$

thereby producing the computable estimation formula

$$
\left|J(u)-J\left(u_{h}\right)\right| \leq \dot{C}_{0} C_{\text {stab }}\left(\sum_{n=0}^{N-1} \sum_{Q^{n}}\left(h^{s}\left\|r_{h}\right\|_{Q^{n}}+h^{s-1 / 2}\left\|j_{h}\right\|_{\partial Q^{n}}\right)^{2}\right)^{1 / 2} .
$$

The basis idea is that it may be feasible to catalog stability constants $C_{\text {stab }}$ for various flow problems so that computation of the dual problem can be avoided. The simplifying assumptions utilized here lead to an unavoidable loss in sharpness when compared to the weighted error estimate.

Next, we briefly review the relevant theory for representing the space-time error in functionals which serves as the primogenitor for both of the previously given a-posteriori estimates.

\section{Error Estimation of Functionals}

Consider once again the model space-time problem (4) in a domain $\Omega \subset \mathbb{R}^{d}$ with boundary $\Gamma$. In this domain, let $\mathcal{V}_{h, p}^{\mathrm{B}}$ denote a mesh dependent broken space of piecewise polynomials of complete degree $p$ in each $Q^{n}$ with no continuity between space-time elements

$$
\mathcal{V}_{h, p}^{\mathrm{B}}=\left\{w:\left.w\right|_{Q} \in \mathcal{P}_{p}(Q), \forall Q \in \mathcal{K} \times \cup_{n=0}^{N-1} I^{n}\right\} .
$$

To illustrate the abstract framework for error estimation of functionals, we consider the discontinuous Galerkin (DG) finite element method introduced by Reed and Hill [24] and LeSaint and Raviart [19] as analyzed by Johnson and Pitkäranta [16] and further refined for nonlinear conservation laws by Cockburn et al. $[4,5]$. In presenting the abstract theory, one quickly sees that the techniques used here can be easily applied to any method that can be written in weighted residual form.

Space-Time Discontinuous Galerkin FEM. Find $u_{h} \in \mathcal{V}_{h, p}^{\mathrm{B}}$ such that

$$
\mathcal{B}_{\mathrm{DG}}\left(u_{h}, w\right)=F(w), \quad \forall w \in \mathcal{V}_{h, p}^{\mathrm{B}}
$$

where 


$$
\begin{aligned}
\mathcal{B}_{\mathrm{DG}}\left(u_{h}, w\right)-F(w)= & \sum_{n=0}^{N-1}\left(\sum _ { Q ^ { n } } \left(\int_{Q^{n}}\left(-w_{, t} \cdot u_{h}-w_{, x_{i}} \cdot f_{i}\left(u_{h}\right)\right) d x d t\right.\right. \\
& +\int_{I^{n}} \int_{\left(\partial K \cap \partial Q^{n}\right) \backslash \Gamma} w_{-} \cdot h\left(n ;\left(u_{h}\right)_{-},\left(u_{h}\right)_{+}\right) d s d t \\
& \left.+\int_{I^{n}} \int_{\left(\partial K \cap \partial Q^{n}\right) \cap \Gamma}^{w_{-}} \cdot h\left(n ;\left(u_{h}\right)_{-}, g\right) d s d t\right) \\
& +\int_{\Omega, n \neq 0}\left(w\left(t_{-}^{n+1}\right) u_{h}\left(t_{-}^{n+1}\right)-w\left(t_{+}^{n}\right) u_{h}\left(t_{-}^{n}\right)\right) d x \\
& \left.\left.+\int_{\Omega, n=0}\left(w\left(t_{-}^{1}\right) u_{h}\left(t_{-}^{1}\right)-w\left(t_{+}^{0}\right) u_{0}\right)\right) d x\right)
\end{aligned}
$$

where $h\left(n ; u_{-}, u_{+}\right)$is a numerical flux function such that $h(n ; u, u)=n_{i} f_{i}(u)$ and $h\left(n ; u_{-}, u_{+}\right)=-h\left(-n ; u_{+}, u_{-}\right)$.

\subsection{DG FEM Error Representation: The Linear Case}

The objective is to estimate the error in a user specified functional $J(u)$, the mapping of a function space to a single real number. In this work, we consider functionals that can be expressed as a weighted integration over the space-time domain

$$
J_{\psi}(u)=\int_{0}^{T} \int_{\Omega} \psi \cdot N(u) d x d t
$$

or a weighted integration on the boundary of the space-time domain

$$
J_{\psi}(u)=\int_{\partial(\Omega \times[0, T])} \psi \cdot N(u) d s
$$

for some user specified weighting function $\psi(x): \mathbb{R}^{d} \mapsto \mathbb{R}^{m}$ and linear/nonlinear function $N(u): \mathbb{R}^{m} \mapsto \mathbb{R}^{m}$. By an appropriate choice of $\psi(x)$ and $N(u)$, it is possible to devise functionals of practical engineering use, e.g. lift and drag forces on a body, stress intensity factors, average quantities, etc.

Let $B_{\mathrm{DG}}(\cdot, \cdot)$ denote a bilinear form for the discontinuous Galerkin method and $J(\cdot)$ a linear functional. In the following derivations, $\pi_{h}$ denotes any suitable projection operator (e.g. interpolation, $L_{2}$ projection) into $\mathcal{V}_{h, p}^{\mathrm{B}}$. Begin by introducing the primal numerical method assuming all boundary conditions are weakly enforced.

Primal numerical problem: Find $u_{h} \in \mathcal{V}_{h, p}^{\mathrm{B}}$ such that

$$
B_{\mathrm{DG}}\left(u_{h}, w\right)=F(w) \quad \forall w \in \mathcal{V}_{h, p}^{\mathrm{B}}
$$

with the Galerkin orthogonality condition

$$
B_{\mathrm{DG}}\left(u-u_{h}, w\right)=0 \quad \forall w \in \mathcal{V}_{h, p}^{\mathrm{B}}
$$

Next, we introduce the auxiliary dual problem utilizing infinite-dimensional trial and test spaces.

Dual problem: Find $\Phi \in \mathcal{V}^{\mathrm{B}}$ such that

$$
B_{\mathrm{DG}}(w, \Phi)=J(w) \quad \forall w \in \mathcal{V}^{\mathrm{B}} .
$$


An exact error representation formula for a given functional $J(\cdot)$ results from the following steps

$$
\begin{aligned}
J(u)-J\left(u_{h}\right) & =J\left(u-u_{h}\right) & & \text { (linearity of } M) \\
& =B_{\mathrm{DG}}\left(u-u_{h}, \Phi\right) & & \text { (dual problem) } \\
& =B_{\mathrm{DG}}\left(u-u_{h}, \Phi-\pi_{h} \Phi\right) & & \text { (orthogonality) } \\
& =B_{\mathrm{DG}}\left(u, \Phi-\pi_{h} \Phi\right)-B_{\mathrm{DG}}\left(u_{h}, \Phi-\pi_{h} \Phi\right) & & \text { (linearity of } B) \\
& =F\left(\Phi-\pi_{h} \Phi\right)-B_{\mathrm{DG}}\left(u_{h}, \Phi-\pi_{h} \Phi\right) & & \text { (primal problem) }
\end{aligned}
$$

so in summary

$$
J(u)-J\left(u_{h}\right)=F\left(\Phi-\pi_{h} \Phi\right)-B_{\mathrm{DG}}\left(u_{h}, \Phi-\pi_{h} \Phi\right) .
$$

Notably absent from the right-hand side of this equation is any dependence on the exact solution $u$.

\subsection{DG FEM Error Representation: The Nonlinear Case}

Let $\mathcal{B}_{\mathrm{DG}}(\cdot, \cdot)$ denote a semilinear form for the discontinuous Galerkin method and $J(\cdot)$ now a nonlinear functional. To cope with nonlinearity, mean-value linearization is employed

$$
\begin{aligned}
\mathcal{B}_{\mathrm{DG}}(u, w) & =\dot{\mathcal{B}}_{\mathrm{DG}}\left(u_{h}, w\right)+\overline{\mathcal{B}}_{\mathrm{DG}}\left(u_{h}, u ; u-u_{h}, w\right) \quad \forall w \in \mathcal{V}^{\mathrm{B}} \\
J(u) & =J\left(u_{h}\right)+\bar{J}\left(u_{h}, u ; u-u_{h}\right) .
\end{aligned}
$$

For example, if $\mathcal{B}(u, w)=(\mathcal{L} u, w)$ for some nonlinear differential operator $\mathcal{L}$ then for $w \in \mathcal{V}$

$$
\begin{aligned}
\mathcal{B}(u, w) & =\mathcal{B}\left(u_{h}, w\right)+\left(\int_{0}^{1} \mathcal{L}_{, u}(\tilde{u}(\theta)) d \theta\left(u-u_{h}\right), v\right) \\
& =\mathcal{B}\left(u_{h}, w\right)+\left(\overline{\mathcal{L}}_{, u}\left(u-u_{h}\right), w\right) \\
& =\mathcal{B}\left(u_{h}, w\right)+\overline{\mathcal{B}}\left(u_{h}, u ; u-u_{h}, w\right) .
\end{aligned}
$$

with $\tilde{u}(\theta) \equiv u_{h}+\left(u-u_{h}\right) \theta$. For brevity, the dependence of $\overline{\mathcal{B}}$ on the path integration involving the exact solution $u$ will be notationally suppressed. We then proceed in the same fashion as in the previous example.

Primal numerical problem: Find $u_{h} \in \mathcal{V}_{h, p}^{\mathrm{B}}$ such that

$$
\mathcal{B}_{\mathrm{DG}}\left(u_{h}, w\right)=F(w) \quad \forall w \in \mathcal{V}_{h, p}^{\mathrm{B}}
$$

with orthogonality condition for the linearized form

$$
\overline{\mathcal{B}}_{\mathrm{DG}}\left(u-u_{h}, w\right)=0 \quad \forall w \in \mathcal{V}_{h, p}^{\mathrm{B}} .
$$

A mean-value linearized dual problem is then introduced which utilizes infinitedimensional trial and test spaces.

Linearized dual problem: Find $\Phi \in \mathcal{V}^{\mathrm{B}}$ such that

$$
\overline{\mathcal{B}}_{\mathrm{DG}}(w, \Phi)=\bar{J}(w) \quad \forall w \in \mathcal{V}^{\mathrm{B}}
$$


An exact error representation formula for a given nonlinear functional $J(\cdot)$ then results from the following steps

$$
\begin{aligned}
J(u)-J\left(u_{h}\right) & =\bar{J}\left(u-u_{h}\right) & & \text { (mean-value } \bar{J}) \\
& =\overline{\mathcal{B}}_{\mathrm{DG}}\left(u-u_{h}, \Phi\right) & & \text { (dual problem) } \\
& =\overline{\mathcal{B}}_{\mathrm{DG}}\left(u-u_{h}, \Phi-\pi_{h} \Phi\right) & & \text { (orthogonality) } \\
& =\mathcal{B}_{\mathrm{DG}}\left(u, \Phi-\pi_{h} \Phi\right)-\mathcal{B}_{\mathrm{DG}}\left(u_{h}, \Phi-\pi_{h} \Phi\right) & & \text { (mean-value } \overline{\mathcal{B}}) \\
& =F\left(\Phi-\pi_{h} \Phi\right)-\mathcal{B}_{\mathrm{DG}}\left(u_{h}, \Phi-\pi_{h} \Phi\right), & & \text { (primal problem) }
\end{aligned}
$$

so in summary

$$
J(u)-J\left(u_{h}\right)=F\left(\Phi-\pi_{h} \Phi\right)-\mathcal{B}_{\mathrm{DG}}\left(u_{h}, \Phi-\pi_{h} \Phi\right) .
$$

Note that although Eqns. (10) and (15) appear identical, mean-value linearization introduces a subtle right-hand side dependency on the exact solution in Eqn. (15).

\section{Computable Error Estimates and Adaptivity}

Computationally, the error representation formulas (10) and (15) are not suitable for obtaining computable a posteriori error estimates and use in mesh adaptation.

- The function $\Phi-\pi_{h} \Phi$ is unknown where $\Phi \in \mathcal{V}^{B}$ is a solution of the infinitedimensional dual problem.

- The mean-value linearization used in the linearized dual problems (14) requires knowledge of the exact solution $u$.

Various strategies which address the numerical approximation of $\Phi$ are discusised in Barth and Larson [2], e.g. postprocessing, higher order solves, etc. Due to Galerkin orthogonality, the dual problem in the discontinuous Galerkin finite element method must be approximated in a larger space-of functions than that utilized in the primal numerical problem. For purposes of the present study, this is achieved in the discontinuous Galerkin method by solving the dual problem using a polynomial space that is one polynomial degree higher than the primal numerical problem, viz. if $u_{h} \in \mathcal{V}_{h, p}^{\mathrm{B}}$ then $\Phi \approx \Phi_{h} \in \mathcal{V}_{h, p+1}^{\mathrm{B}}$.

In the present study, the mean-value linearization depending on the states $u$ and $u_{h}$ is replaced by the simpler Jacobian linearization evaluated at the numerical state $u_{h}$. This is not the only practical choice. In Barth and Larson [2], a more sophisticated technique involving the postprocessing of primal data and the approximation of the mean-value linearization by numerical quadrature is employed in computations.

\subsection{Direct Estimates}

When written in global abstract form, the error representation formula does not indicate which elements in the mesh should be refined to reduce the measured error in a functional. By applying a sequence of direct estimates, error bounds suitable for adaptive meshing are easily obtained. The goal in constructing these estimates is to estimate the local contribution of each element in the mesh to the functional 
error. This local cell contribution will then be used as an error indicator for choosing which elements to refine or coarsen in the adaptive mesh procedure.

$$
\begin{aligned}
\mid J(u)- & J\left(u_{h}\right)|=| \mathcal{B}_{\mathrm{DG}}\left(u_{h}, \Phi-\pi_{h} \Phi\right)-F\left(\Phi-\pi_{h} \Phi\right) \mid \quad \text { (error representation) } \\
& =\left|\sum_{n=0}^{N-1} \sum_{Q^{n}}\left(\mathcal{B}_{\mathrm{DG}, Q^{n}}\left(u_{h}, \Phi-\pi_{h} \Phi\right)-F_{Q^{n}}\left(\Phi-\pi_{h} \Phi\right)\right)\right| \quad \text { (element assembly) } \\
& \leq \sum_{n=0}^{N-1} \sum_{Q^{n}}\left|\left(\mathcal{B}_{\mathrm{DG}, Q^{n}}\left(u_{h}, \Phi-\pi_{h} \Phi\right)-F_{Q^{n}}\left(\Phi-\pi_{h} \Phi\right)\right)\right| \quad \text { (triangle inequality) }
\end{aligned}
$$

where $\mathcal{B}_{\mathrm{DG}, Q^{n}}(\cdot, \cdot)$ and $F_{Q^{n}}(\cdot)$ are restrictions of $\mathcal{B}_{\mathrm{DG}}(\cdot, \cdot)$ and $F(\cdot)$ to the partition element $Q^{n}$. The basic definition of the discontinuous Galerkin semilinear form given in (10) shows one possible element assembly form but this is not a unique representation. For example strong and weak forms of the semilinear operator $B_{\mathrm{DG}}(\cdot, \cdot)$ yield differing assembly representations. For the discontinuous Galerkin method, the error representation formula together with (10) for a single element $Q^{n}$ yields

$$
\begin{aligned}
\mathcal{B}_{\mathrm{DG}, Q^{n}}\left(u_{h}, \Phi-\pi_{h} \Phi\right)-F_{Q^{n}}\left(\Phi-\pi_{h} \Phi\right) & =-\int_{I^{n}} \int_{K} u_{h} \cdot\left(\Phi-\pi_{h} \Phi\right)_{, t} d x d t \\
& -\int_{I^{n}} \int_{K} f_{i}\left(u_{h}\right) \cdot\left(\Phi-\pi_{h} \Phi\right)_{, x_{i}} d x d t \\
& +\int_{I^{n}} \int_{\partial K \backslash \Gamma}\left(\Phi-\pi_{h} \Phi\right)_{-} \cdot h\left(n ;\left(u_{h}\right)_{-},\left(u_{h}\right)_{+}\right) d s d t \\
& +\int_{I^{n}} \int_{\partial K \cap \Gamma}\left(\Phi-\pi_{h} \Phi\right)_{-} \cdot h\left(n ;\left(u_{h}^{\prime}\right)_{-}, g\right) d s d t \\
& +\int_{K}\left(\Phi-\pi_{h} \Phi\right)\left(t_{-}^{n+1}\right) \cdot u\left(t_{-}^{n+1}\right) d x \\
& -\int_{K, n \neq 0}\left(\Phi-\pi_{h} \Phi\right)\left(t_{+}^{n}\right) \cdot u\left(t_{-}^{n}\right) d x \\
& \left.-\int_{K, n=0}\left(\Phi-\pi_{h} \Phi\right)\left(t_{+}^{0}\right) \cdot u_{0}\right) d x
\end{aligned}
$$

or a weighted residual (strong) form can be obtained upon integration by parts

$$
\begin{aligned}
\mathcal{B}_{\mathrm{DG}, Q^{n}}\left(u_{h}, \Phi-\pi_{h} \Phi\right) & -\dot{F}_{Q^{n}}\left(\Phi-\pi_{h} \Phi\right)=\int_{Q^{n}}\left(\Phi-\pi_{h} \Phi\right) \cdot \mathcal{L} u_{h} d x d t \\
& +\int_{I^{n}} \int_{\partial K \backslash \Gamma}\left(\Phi-\pi_{h} \Phi\right)_{-} \cdot\left(h\left(n ;\left(u_{h}\right)_{-},\left(u_{h}\right)_{+}\right)-f\left(n ;\left(u_{h}\right)_{-}\right) d s d t\right. \\
& +\int_{I^{n}} \int_{\partial K \cap \Gamma}\left(\Phi-\pi_{h} \Phi\right)_{-} \cdot\left(h\left(n ;\left(u_{h}\right)_{-}, g\right)-f\left(n ;\left(u_{h}\right)_{-}\right) d s d t\right) \\
& +\int_{K, n \neq 0}\left(\Phi-\pi_{h} \Phi\right)\left(t_{+}^{n}\right) \cdot[u]_{t_{-}^{n}}^{t_{+}^{n}} d x \\
& +\int_{K, n=0}\left(\Phi-\pi_{h} \Phi\right)\left(t_{+}^{0}\right) \cdot\left(u_{h}\left(t_{+}^{0}\right)-u_{0}\right) d x
\end{aligned}
$$

This latter weighted residual form $\sum_{Q^{n}} \mathcal{B}_{Q^{n}}(\cdot, \cdot)-F_{Q^{n}}(\cdot)$ is preferred in the error estimates (16) since the individual terms represent residual components that vanish individually when the exact solution is inserted into the variational form and a slightly sharper approximation is obtained after application of the triangle inequality in (16). 


\subsection{Adaptive Meshing}

Motivated by the direct estimates (16), we define for each partition element $Q^{n}$ the space-time element quantity

$$
\eta_{Q^{n}} \equiv \mathcal{B}_{\mathrm{DG}, Q^{\mathrm{n}}}\left(u_{h}, \Phi-\pi_{h} \Phi\right)-F_{Q^{n}}\left(\Phi-\pi_{h} \Phi\right)
$$

so that $\left|\eta_{Q^{n}}\right|$ serves as an adaptation element indicator such that

$$
\left|J(u)-J\left(u_{h}\right)\right| \leq \sum_{n=0}^{N-1} \sum_{Q^{n}}\left|\eta Q^{n}\right|
$$

and the sum of $\eta_{Q^{n}}$ over space-time provides an accurate adaptation stopping criteria

$$
\left|J(u)-J\left(u_{h}\right)\right|=\left|\sum_{n=0}^{N-1} \sum_{Q^{n}} \eta_{Q^{n}}\right| .
$$

These quantities suggest a simple mesh adaptation strategy in common use with other indicator functions:

Mesh Adaptation Algorithm

(1) Construct an initial space-time mesh.

(2) Compute a numerical approximation of the primal problem on the current spacetime mesh.

(3) Compute a suitable numerical approximation of the infinite-dimensional dual problem on the current mesh.

(4) Compute error indicators, $\eta_{Q^{n}}$.

(5) If $\left(\left|\sum_{n=0}^{N-1} \sum_{Q^{n}} \eta_{Q^{n}}\right|<T O L\right)$ STOP

(6) Otherwise, refine and coarsen a specified fraction of the total number of elements according to the size of $|\eta|_{Q}^{n}$, generate a new mesh and GOTO 2

\subsection{Numerical Example: Error Estimates for Time-Dependent Hyperbolic Problems}

Numerical error estimation results for stationary hyperbolic problems using the discontinuous Galerkin method and the error estimates described above are given by the present author in $[18,2]$ and later revisited by Hartman and Houston in [13]. To demonstrate the application of the error representation formula (10) and the error bound (16) to time-dependent hyperbolic problems, discontinuous Galerkin solutions have been obtained for the following 2-D advection problem in the square domain $\Omega \in[-1,1]^{2}$

$$
\left\{\begin{aligned}
u_{, t}+\lambda \cdot \nabla u & =0 & & \text { in } \Omega \times[0, T] \\
u(x, t) & =0 & & \text { on } \partial \Omega \times[0, T] \\
u(x, 0) & =u_{0}(x) & & \text { initial data }
\end{aligned}\right.
$$

with circular advection field $\lambda=(-y, x)^{T}$, final time $T=1.15$, and $C^{\infty}$ initial data

$$
u_{0}(x)=\sigma\left(2 / 10 ; \sqrt{\left(x-x_{0}\right)^{2}+\left(y-y_{0}\right)^{2}}\right), \quad\left(x_{0}, y_{0}\right)=(7 / 10,0)
$$


with

$$
\sigma\left(r_{0} ; r\right) \equiv\left\{\begin{array}{cc}
0 & r \geq r_{0} \\
e^{1+r_{0}^{2} /\left(r^{2}-r_{0}^{2}\right)} & r<r_{0}
\end{array} .\right.
$$

An output functional was also devised consisting of a $C^{\infty}$ space-time averaging ball of radius $1 / 4$ located at $\left(x_{f}, y_{f}, t_{f}\right)=(.30, .26, .64)$, i.e.

$$
J(u)=\int_{0}^{T} \int_{\Omega} \sigma\left(1 / 3 ; \sqrt{\left(t-t_{f}\right)^{2}+\left(x-x_{f}\right)^{2}+\left(y-y_{f}\right)^{2}}\right) d x d t
$$
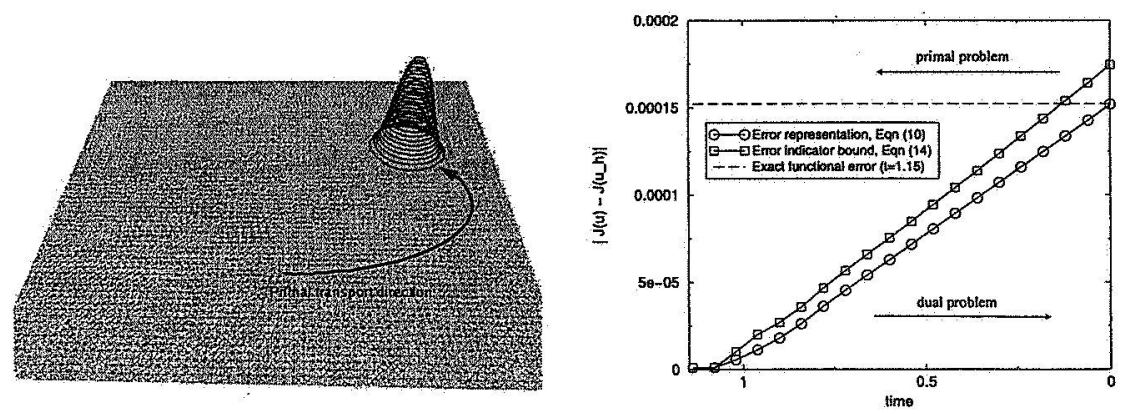

Fig. 2. Primal solution of the circular advection problem at $t=45$. Shown are a carpet plot of the primal solution using linear space-time elements (left) and a graph of the accumulated functional error, $J(u)-J\left(u_{h}\right)$, during the backwards in time dual solution integration (right).
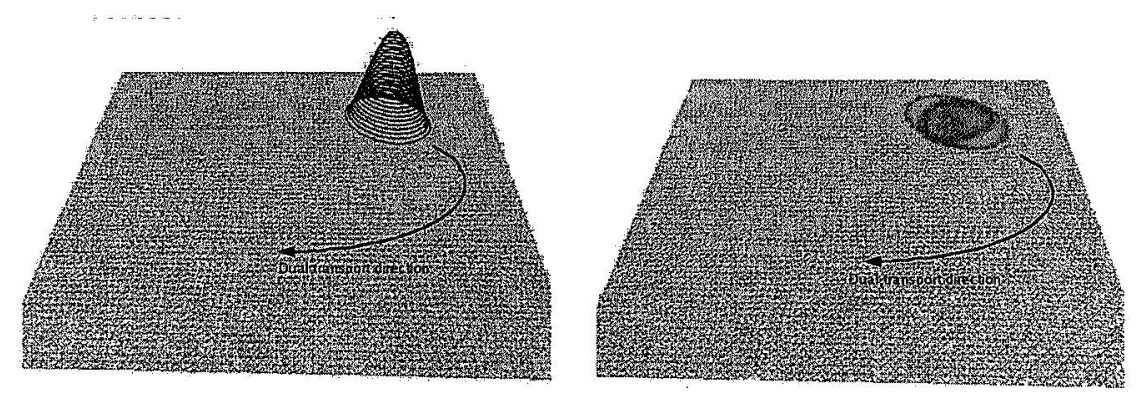

Fig. 3. Dual solution of the circular advection problem at $t=.45$. Shown are a carpet plot of the dual solution $\phi_{h}$ using quadratic space-time elements (left) and a carpet plot of the dual solution defect $\phi_{h}-\pi_{h} \phi_{h}$ (right) with $\pi_{h}$ a projection to space-time linear functions.

The numerical experiment then consists for the following steps 
1. Numerical solution, $u_{h}$, of the primal (forwards in time) problem using the discontinuous Galerkin method (9) with linear space-time elements, see Fig. 2(left).

2. Numerical solution, $\phi_{h}$, of the dual (backwards in time) problem using the discontinuous Galerkin method (9) with quadratic space-time elements, see Fig. 3.

3. Approximation of the infinite-dimensional dual problem by the quadratic spacetime element approximation, $\phi \approx \phi_{h}$.

4. Accumulation of the functional error, $\left|J(u)-J\left(u_{j}\right)\right|$, using the error representation formula (10) and the error bound formula (16), see Fig. 2(right).

Note that although we plot in Fig. 2 the accumulation of terms appearing in the error estimation formulas (10) and (16) during the backwards in time integration, it is only the final termination value at time zero that provides a bound for the desired functional error. As expected, the error representation formula very accurately reproduces the exact functional error for this linear differential equation since the only source of approximation in this formula is the replacement of $\phi$ by a higher order quadratic space-time element approximation $\phi_{h}$. The graph in Fig. 2 corresponding to Eqn. (16) lies strictly above the error representation formula since the absolute value occurring in the Eqn. (16) precludes any interelement cancellation of error terms. In this particular example, this lack of interelement cancellation in Eqn. (16) also gives rise to a different slope and a continuous deterioration in Eqn. 16 with increasing functional measurement time.

\section{Extension to the Time-Dependent Navier-Stokes Equations}

Next, we consider the error estimation of functionals applied to the time-dependent compressible Navier-Stokes equations for a perfect gas with fluid density, velocity, pressure, and temperature denoted by $\rho, V, p$ and $T$

$$
u_{, t}+F_{i, x_{i}}^{\mathrm{inv}}=F_{i, x_{i}}^{\mathrm{vis}} \text { in } \Omega \times[0, T]
$$

with implied summation on repeated indices, $1, \ldots, d$, and

$$
\begin{gathered}
u=\left(\begin{array}{c}
\rho \\
\rho V_{1} \\
\vdots \\
\rho V_{d} \\
\rho E
\end{array}\right), \quad F_{i}^{\mathrm{inv}}=\left(\begin{array}{c}
\rho V_{i} \\
\rho V_{1}+\delta_{i 1} p \\
\vdots \\
\rho V_{i} V_{d}+\delta_{i d} p \\
V_{i}(E+p)
\end{array}\right), \quad F_{i}^{\mathrm{vis}}=\frac{1}{R e}\left(\begin{array}{c}
0 \\
\tau_{1 i} \\
\vdots \\
\tau_{3 i} \\
\tau=\frac{\mu}{R e}\left(\nabla V+(\nabla V)^{T}-\frac{2}{3}(\nabla \cdot V) I\right)
\end{array}\right.
\end{gathered}
$$

where $\gamma, R e$, and $\operatorname{Pr}$ denote the non-dimensional ratio of specific heats, Reynolds number and Prandtl numbers, respectively.

The present implementation of the discontinuous Galerkin method utilizes a change of dependent variables, $u \mapsto v$, where $u$ are the so-called symmetrization variables for the Navier-Stokes equations as described in [15]. Using this change of variables, viscous fluxes are written in chainrule form 


$$
F_{i}^{\mathrm{vis}}=M_{i j} v_{x_{j}}, \quad M_{i j} \in \mathbb{R}^{m \times m} .
$$

The extension of the discontinuous Galerkin method to the time-dependent NavierStokes equations follows the original symmetric interior penalty (SIP) work of Douglas and Dupont [6] for elliptic and parabolic problems. The SIP formulation has been recently applied to the steady Navier-Stokes equations in $[11,12]$. The present SIP formulation and subsequent error estimation calculations accommodates the full time-dependent compressible Navier-Stokes equations. For brevity, the method is stated here in strong form. In practice, the method should always be implemented in weak integrated-by-parts form so that exact discrete conservation is assured even in the presence of inexact numerical quadrature.

Space-Time Discontinuous Galerkin SIP Navier-Stokes FEM. Find $v_{h} \in \mathcal{V}_{h, p}^{\mathrm{B}}$ such that

$$
\sum_{n=0}^{N-1} \sum_{K} B_{\mathrm{DG}, Q^{n}}\left(v_{h}, w\right)=0, \quad \forall w \in \mathcal{V}_{h, p}^{\mathrm{B}}
$$

with

$$
\begin{aligned}
& B_{\mathrm{DG}, Q^{n}}(v, w)=\int_{K} \int_{I^{n}} w \cdot\left(u_{, t}+F_{i, x_{i}}^{\mathrm{inv}}-F_{i, x_{i}}^{\mathrm{vis}}\right) d t d x \\
& +\int_{\partial K \backslash \Gamma} \int_{I^{n}} w\left(x_{-}\right) \cdot\left(h\left(n ; v_{+}, v_{-}\right)-n_{i} F_{i}^{\mathrm{inv}}\left(v_{-}\right)\right) d t d x \\
& \left.+\int_{\partial K \cap \Gamma_{\text {wall }}} \int_{I^{n}} w\left(x_{-}\right) \cdot n_{i}\left(F_{i}^{\text {inv wall }}-F_{i}^{\text {inv }}\right)\left(v_{-}\right)\right) d t d x \\
& +\int_{\partial K \cap \Gamma_{\text {farfield }}} \int_{I^{n}} w\left(x_{-}\right) \cdot\left(h\left(n ; g_{\infty}, v_{-}\right)-n_{i} F_{i}^{i n v}\left(v_{-}\right)\right) d t d x \\
& -\int_{\partial K \backslash \Gamma} \int_{I^{n}}^{\frac{1}{2}} w\left(x_{-}\right) \cdot\left[n_{i} F_{i}^{\mathrm{vis}}\right]_{x_{-}}^{x_{+}} d t d x \\
& -\int_{\partial K \cap \Gamma_{\mathrm{N}}} \int_{I^{n}} w\left(x_{-}\right) \cdot n_{i}\left(F_{i}^{\mathrm{vis}}\left(g_{N}\right)-F_{i}^{\mathrm{vis}}\left(v_{-}\right)\right) d t d S \\
& +\int_{\partial K \backslash \Gamma} \int_{I^{n}} \frac{1}{2}[v]_{x_{-}}^{x_{+}} \cdot n_{i} M_{i j}\left(x_{-}\right) w_{, x_{j}}\left(x_{-}\right) d t d x \\
& +\int_{\partial K \cap \Gamma_{\mathrm{D}}} \int_{I^{n}}\left(g_{D}-v\left(x_{-}\right)\right) \cdot n_{i} M_{i j}\left(x_{-}\right) w_{, x_{j}}\left(x_{-}\right) d t d x \\
& \left.-\int_{\partial K \backslash \Gamma} \int_{I^{n}}\left\langle\kappa p^{2} / h\right\rangle_{x_{-}}^{x_{+}} w\left(x_{-}\right)\right) \cdot n_{i} n_{j} M_{i j}[v]_{x_{-}}^{x_{+}} d t d x \\
& \left.\left.-\int_{\partial K \cap \Gamma_{\mathrm{D}}} \int_{\Gamma^{n}}\left(\kappa p^{2} / h\right)_{x_{-}} w\left(x_{-}\right)\right) \cdot n_{i} n_{j} M_{i j}\left(g_{D}-v\left(x_{-}\right)\right)\right) d t d x \\
& +\int_{K, n \neq 0} w\left(t_{+}^{n}\right) \cdot[u(v)]_{t_{-}^{n}}^{t_{+}^{n}} d x \\
& +\int_{K, n=0} w\left(t_{+}^{0}\right) \cdot\left(u\left(v\left(t_{-}^{0}\right)\right)-u_{0}\right) d x
\end{aligned}
$$

where $\kappa$ is an $\mathcal{O}(1)$ parameter, $\langle\cdot\rangle_{x_{-}}^{x_{+}} \equiv\left((\cdot)_{x_{-}}+(\cdot)_{x_{+}}\right) / 2$ and $g_{\infty}, g_{N}$ and $g_{D}$ denote farfield, Neumann, and Dirichlet data, respectively. This formulation immediately gives a convenient error representation formula of the same form as (15)

$$
J\left(u\left(v_{h}\right)\right)-J(u(v))=\sum_{n=0}^{N-1} \sum_{K} B_{\mathrm{DG}, Q^{n}}\left(v_{h}, \phi-\pi_{h} \phi\right)
$$


and an error estimate suitable for mesh adaptivity of the same form as (16)

$$
\left|J\left(u\left(v_{h}\right)\right)-J(u(v))\right| \leq \sum_{n=0}^{N-1} \sum_{K}\left|B_{\mathrm{DG}, Q^{n}}\left(v_{h}, \phi-\pi_{h} \phi\right)\right| .
$$

\subsection{Numerical Example: Time-Dependent Compressible Navier-Stokes 2D Cylinder Flow at $R e=300$}

The accurate prediction of lift and drag forces on a cylinder undergoing vortex shedding remains an important and difficult test problem in numerical timedependent Navier-Stokes simulations $[21,8,20]$. In the present study, subsonic flow at $\mathrm{Mach}=1 / 10$ and $\mathrm{Re}=300$ has been computed over a $2 \mathrm{D}$ cylinder for a length of time sufficient to establish periodic vortex shedding. A numerical solution suitable for assessing error estimates and adaptation was computed using $12 K$ linear spacetime elements and a time step size resulting in approximately 48 time steps per oscillation period. Another extremely accurate "reference" solution was also computed using $40 K$ quadratic space-time elements and $50 \%$ reduced timestep, see Fig. 4.
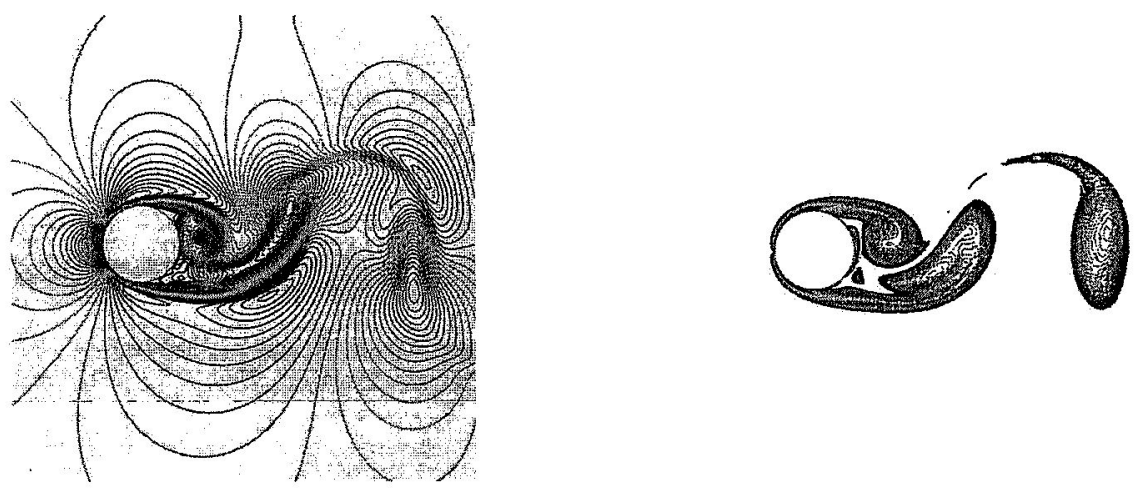

Fig. 4. Navier-Stokes solution at the non-dimensional time $t=635$ computed on the reference $40 \mathrm{~K}$ element mesh using $\mathcal{P}_{2}$ space-time elements. Presented here are velocity contours (left) and logarithmically scaled vorticity magnitude contours (right).

A measurement functional was then devised consisting of the the non-dimensional drag force integrated over the cylinder surface and averaged over four drag oscillation periods, $t \in[605,700]$, determined from the reference solution with time non-dimensionalized here using freestream sound speed and cylinder diameter.

$$
J(u)=\frac{D}{1 / 2 \rho_{\infty} V_{\infty}^{2}} \frac{1}{t_{b}-t_{a}} \int_{t_{\alpha}}^{t_{b}} \int_{\text {cyl wall }} F_{\mathrm{drag}}(u) d x d t .
$$

Following the same procedure as outline earlier in Sect. 3.3, a dual solution corresponding to the time-averaged drag functional was obtained on the $12 K$ mesh using quadratic space-time elements. This solution serves as an approximation of the 
infinite-dimension dual solution $\phi$. Figure 5 provides a time snapshot of the quadratic space-time element dual solution, $\phi_{h}$, and the defect dual solution, $\phi_{h}-\pi_{h} \phi_{h}$, where $\pi_{h}$ is the $L_{2}$ space-time projection from quadratic elements to linear elements. A
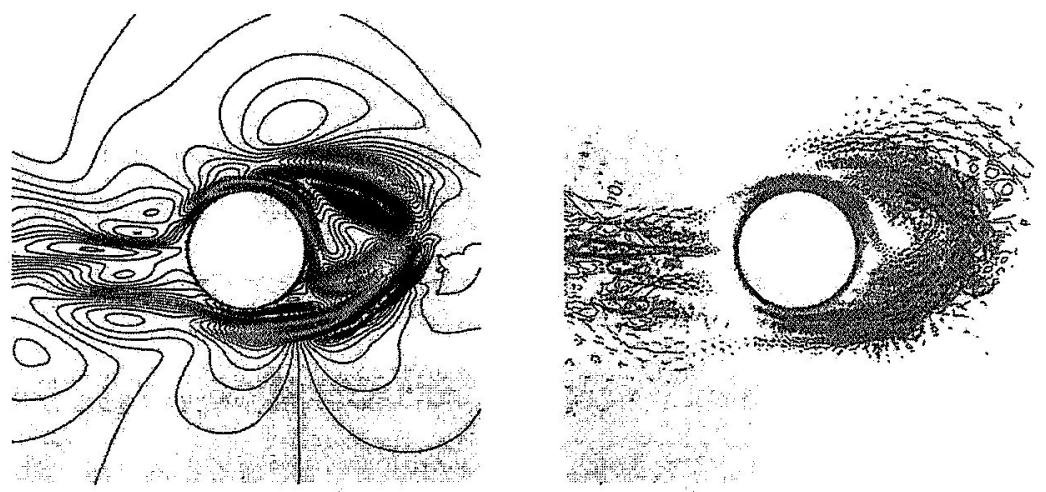

Fig. 5. Locally linearized dual Navier-Stokes solution corresponding to the drag coefficient functional with local linearization about $\mathcal{P}_{1}$ primal space-time data on a $12 \mathrm{~K}$ mesh. Presented here are dual solution contours at the same approximate nondimensional time as Fig. 4 showing the $x$-momentum component of the dual solution $\phi$ (left) and the $\mathrm{x}$-momentum component of the dual solution defect $\phi-\pi_{h} \phi$ (right).

rather striking feature of the dual solution as is illustrated in Fig. 5 is the localized support of the defect $\phi-\pi_{h} \phi$ when compared to $\phi$ itself. Since only the defect $\phi-\pi_{h} \phi$ appears in the error representation formula (28), the resulting mesh adaptivity becomes very localized as well. Although it is possible to adapt the mesh during every time step of the calculation based on error indicators derived from (29), in the present calculations the mesh indicators have been averaged over four oscillation periods so that a single (static) mesh adaptation is performed, see Fig. 6. Figure 7
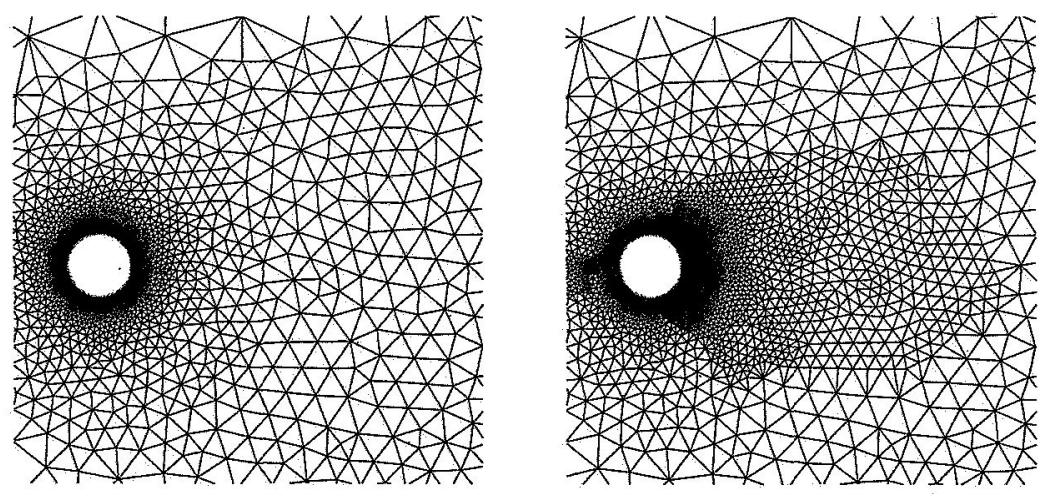

Fig. 6. Closeup of the original $12 K$ element mesh (left) and refined $15 K$ element mesh (right) obtained from error indicators averaged over four oscillation periods. 
shows the lift and drag coefficient histories on all meshes. A comparison of drag coefficient histories shown in this figure reveals a dramatic improvement in oscillation amplitude and mean value with only modest mesh adaptivity. Table 2 quantifies the
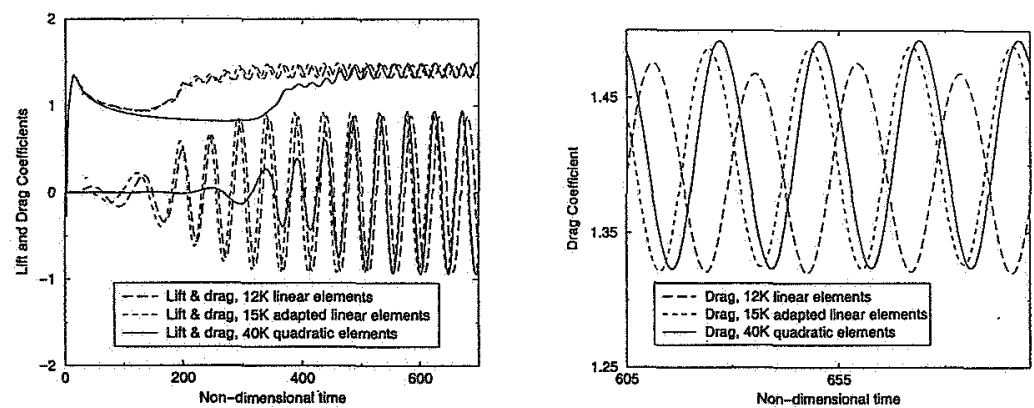

Fig. 7. Lift and drag coefficient history for 2D Navier-Stokes cylinder flow at $\mathrm{Re}=300$ and $\mathrm{Mach}=0.1$ using $12 K \mathcal{P}_{1}$ space-time elements, $15 K \mathcal{P}_{1}$ adapted spacetime elements and $40 K \mathcal{P}_{2}$ space-time elements. Shown are the lift and drag coefficient histories (left) and an extreme closeup of the drag coefficient histories in the functional interval (right).

accuracy of the computed functional error using the $40 \mathrm{~K}$ element reference solution as the "exact" solution together with the estimated values from (28) and (29). The

\begin{tabular}{|c|c|c|c|}
\hline \#elements & $\left|J(u)-J\left(u_{h}\right)\right|$ & Eqn. (28) & Eqn. (29) \\
\hline $12 \mathrm{~K}$ & .0107 & .0183 & .1098 \\
$15 \mathrm{~K}$ (adapted) & .0020 & .0024 & .0139 \\
\hline
\end{tabular}

Table 2. Error estimates for the 2D cylinder on meshes with $12 K$ and $15 K$ adapted elements.

tabulated results show some lack of sharpness in the computed error representation formula (28) that would be further amplified in the estimate (29). The improvement with adaptive mesh refinement may indicate that this source of error originates from linearization error resulting from the use of the jacobian linearization as a replacement for the mean-value linearization in (11) and (12). Another source of error may originate from the specification of the four period averaging interval in the functional definition. These issues will be addressed in future expanded work for this problem.

\section{Concluding Remarks}

As one might expect, the extension of a-posteriori error estimation to space-time is almost transparent for finite element methods that permit discretization in time 
using the same finite element method. Even so, the implied added computation is significant since for time-dependent problems a backwards in time dual problem must be solved or approximated. Many open problems exist in this problem area:

- Efficient techniques for approximating the dual in space-time problem.

- Computable functionals for time-dependent problems that do not lead to dual problems that become large or unbounded in reverse time.

- Techniques for estimating the error in general $L_{p}$ norms.

\section{References}

1. T. J. Barth and H. Deconinck(eds). Error estimation and adaptive discretization methods in CFD. In Barth and Deconinck, editors, Error Estimation and Adaptive Discretization Methods in CFD, volume 25 of Lecture Notes in Computational Science and Engineering. Springer-Verlag, Heidelberg, 2002.

2. T. J. Barth and M.G. Larson. A-posteriori error estimation for higher order Godunov finite volume methods on unstructured meshes. In Herbin and Kröner, editors, Finite Volumes for Complex Applications III, pages 41-63. Hermes Science Pub., London, 2002.

3. R. Becker and R. Rannacher. Weighted a posteriori error control in FE methods. In Proc. ENUMATH-97, Heidelberg. World Scientific Pub., Singapore, 1998.

4. B. Cockburn, S.Y. Lin, and C.W. Shu. TVB Runge-Kutta local projection discontinuous Galerkin finite element method for conservation laws III: One dimensional systems. J. Comp. Phys., 84:90-113, 1989.

5. B. Cockburn and C.W. Shu. The Runge-Kutta discontinuous Galerkin method for conservation laws V: Multidimensional systems. Technical Report 201737, Institute for Computer Applications in Science and Engineering (ICASE), NASA Langley R.C., 1997.

6. J. Douglas and T. Dupont. Interior penalty procedures for elliptic and parabolic

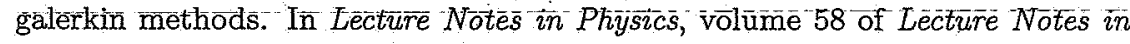
Physics. Springer-Verlag, Heidelberg, 1976.

7. K. Eriksson, D. Estep, P. Hansbo, and C. Johnson. Introduction to numerical methods for differential equations. Acta Numerica, pages 105-158, 1995.

8. C. Farell and J. Blessman. On critical flow around smooth circular cylinders. J. Fluid Mech., 136, 1983.

9. M. Giles, M. Larson, M. Levenstam, and E. Süli. Adaptive error control for finite element approximations of the lift and drag coefficients in viscous flow. preprint NA-97/06, Comlab, Oxford University, 1997.

10. M. Giles and N.A. Pierce. Improved lift and drag estimates using adjoint Euler equations. Technical Report 99-3293, AIAA, Reno, NV, 1999.

11. R. Hartman and P. Houston. Symmetric interior penalty DG methods for the compressible Navier-Stokes equations I: Method formulation. Int. J. Numer. Anal. Model., to appear.

12. R. Hartman and P. Houston. Symmetric interior penalty DG methods for the compressible Navier-Stokes equations II: Goal-oriented a posteriori error estimation. Int. J. Numer. Anal. Model., to appear.

13. R. Hartmann and P. Houston. Adaptive discontinuous Galerkin methods for the compressible euler equations. J. Comp. Phys., 182(2):508-532, 2002. 
14. J. Hoffman and C. Johnson. Adaptive finite element methods in incompressible fluid flow. In Barth and Deconinck, editors, Error Estimation and Adaptive Discretization Methods in CFD, volume 25 of Lecture Notes in Computational Science and Engineering. Springer-Verlag, Heidelberg, 2002.

15. T. J. R. Hughes, L. P. Franca, and M. Mallet. A new finite element formulation for CFD: I. symmetric forms of the compressible Euler and Navier-Stokes equations and the second law of thermodynamics. Comp. Meth. Appl. Mech. Engrg., 54:223-234, 1986.

16. C. Johnson and J. Pitkäranta. An analysis of the discontinuous Galerkin method for a scalar hyperbolic equation. Math. Comp., 46:1-26, 1986.

17. C. Johnson, R. Rannacher, and M. Boman. Numerics and hydrodynamics stability theory: towards error control in CFD. SIAM J. Numer. Anal., 32:1058-1079, 1995.

18. M.G. Larson and T.J. Barth. A posteriori error estimation for adaptive discontinuous Galerkin approximations of hyperbolic systems. In Cockburn, Karniadakis, and Shu, editors, Discontinuous Galerkin Methods, volume 11 of Lecture Notes in Computational Science and Engineering. Springer-Verlag, Heidelberg, 1999.

19. P. LeSaint and P.A. Raviart. On a finite element method for solving the neutron transport equation. In C. de Boor, editor, Mathematical Aspects of Finite Elements in Partial Differential Equations, pages 89-145. Academic Press, 1974.

20. R. Mittal and S. Balachandar. Effect of three-dimensionality of the lift and drag on nominally two-dimensional cylinders. Physics of Fluids, 7(8):1841-1865, 1995.

21. C. Norberg. Effects of Reynolds Number and Low-Intensity Free-Stream Turbulence on the Flow Around Circular Cylinders. PhD thesis, Department of Applied Thermosciences and Fluid Mechanics, Chamers University of Technology, Sweden, 1987.

22. J. T. Oden and S. Prudhomme. Goal-oriented error estimation and adaptivity for the finite element method. Technical Report 99-015, TICAM, U. Texas, Austin,TX, 1999.

23. S. Prudhomme and J.T. Oden. On goal-oriented error estimation for elliptic problems: application to the control of pointwise errors. Comp. Meth. Appl. Mech. and Eng., pages 313-331, 1999.

24. W. H. Reed and T. R. Hill. Triangular mesh methods for the neutron transport equation. Technical Report LA-UR-73-479, Los Alamos National Laboratory, Los Alamos, New Mexico, 1973.

25. E. Süli. A posteriori error analysis and adaptivity for finite element approximations of hyperbolic problems. In Kröner, Ohlberger, and Rohde, editors, $A n$ Introduction to Recent Developments in Theory and Numerics for Conservation Laws, volume 5 of Lecture Notes in Computational Science and Engineering, pages 122-194. Springer-Verlag, Heidelberg, 1998. 